\title{
Functional Nanopores: A Solid- state Concept for Artificial Reaction Compartments and Molecular Factories
}

\author{
Gabriel Puebla-Hellmann ${ }^{\star a b}$, Marcel Mayor ${ }^{\mathrm{a}}$, and Emanuel Lörtscher
}

\begin{abstract}
On the road towards the long-term goal of the NCCR Molecular Systems Engineering to create artificial molecular factories, we aim at introducing a compartmentalization strategy based on solid-state silicon technology targeting zeptoliter reaction volumes and simultaneous electrical contact to ensembles of well-oriented molecules. This approach allows the probing of molecular building blocks under a controlled environment prior to their use in a complex molecular factory. Furthermore, these ultra-sensitive electrical conductance measurements allow molecular responses to a variety of external triggers to be used as sensing and feedback mechanisms. So far, we demonstrate the proof-of-concept by electrically contacting selfassembled mono-layers of alkane-dithiols as an established test system. Here, the molecular films are laterally constrained by a circular dielectric confinement, forming a so-called 'nanopore'. Device yields above $85 \%$ are consistently achieved down to sub-50 nm nanopore diameters. This generic platform will be extended to create distributed, cascaded reactors with individually addressable reaction sites, including interconnecting microfluidic channels for electrochemical communication among nanopores and sensing sites for reaction control and feedback. In this scientific outlook, we will sketch how such a solid-state nanopore concept can be used to study various aspects of molecular compounds tailored for operation in a molecular factory.
\end{abstract}

Keywords: Bio-mimicking · Cell · Compartmentalization · Feedback · Molecular Factories · Molecular Transport $\cdot$ Sensing

\section{Introduction and Motivation}

\subsection{The Vision of the National Center for Competence in Research on Molecular Systems Engineering (NCCR MSE)}

The National Center for Competence in Research in Molecular Systems Engineering (NCCR MSE) started in 2014 and represents a highly interdisciplinary project involving life sciences, chemistry, physics and biology as well as bio-informatics and engineering sciences. Its scientific goals aim at synthesizing, assembling and engineering molecular modules, as well as using them to create artificial molecular factories with the long-term goal of

${ }^{*}$ Correspondence: Dr. G. Puebla-Hellmann ${ }^{\text {ab }}$ E-mail: gpu@zurich.ibm.com

aDepartment of Chemistry

University of Basel

St. Johanns-Ring 19, CH-4056 Basel

bIBM Research - Zurich

Säumerstrasse 4, CH-8803 Rüschlikon achieving similar complexities as found in biological systems. Such molecular factories should finally enable unprecedented control over reactions down to the singlemolecule level and establish artificial cellular systems that can be used in industrial production as well as in medical and biological sciences. The envisioned factories require cascades of different and sometimes interlinked tasks and functions, allowing for the synthesis of value-added products in a complex process.

We aim at using a compartmentalization strategy inspired by nature to isolate the individual components in compartments where they can be studied separately under controlled conditions. By connecting different compartments both electrically and electrochemically, this strategy can be expanded as a model to study feedback mechanisms and ultimately used for creating completely artificial systems. Similar to integrated circuits in electronics, these molecular factories will cascade and connect components with different functionalities. While this system can be used to mimic, isolate and study bio-related reactions, a solid-state architecture based on silicon and compatible with standard semi-conductor fabrication will furthermore enable the study and fabrication of products which are bio-incompatible or which require bio-incompatible process parameters.

\subsection{Compartmentalization in Nature}

In biology, functional subunits within a cell are organized in organelles which are separated from each other by a lipid bilayer membrane enclosure, a strategy that is referred to as "cellular compartmentalization'. With only a few exceptions, most organelles fall into this category of cellular compartments: the cell's nucleus, mitochondria, chloroplasts, peroxisomes, lysosomes, the endoplasmic reticulum, etc. Even smaller subunits like vesicles or microtubules can be considered 'cellular compartments' either owing to their functional behavior or their spatial arrangement. Nature's strategy for compartmentalization is based on the following three motives:

- Creating physical boundary conditions for processes that enable various (metabolic) activities to be performed simultaneously

- Generating a compartment-specific micro-environment to initiate, drive and stop processes

- Establishing intracellularly addressable reaction volumes where reactions occur and can be controlled via feedback mechanisms

The spatial isolation created by a compartment on the one hand enables a complex process to be divided into a series of simpler steps, each with differ- 
ent well-defined conditions. On the other hand, compartmentalization allows for a reaction's starting materials and products to be selected in a deterministic way and selectively released after the reaction has taken place. Fig. 1A and B schematically illustrate the control achieved over reactants by using a compartmentalization strategy. The main components required to establish a compartment with a physical boundary, selective in- and outlets as well as a micro-environment are depicted in Fig. 1C.

\section{Artificial Molecular Factories}

\subsection{Bio-inspired Molecular Factories}

Given the evolutionary very successful approach of nature to compartmentalize, the idea of creating artificial reaction compartments to mimic cellular (metabolic) activities down to the few-molecule level seems to be a potential pathway to realize complex molecular factories. However, this concept involves several very challenging tasks and mechanisms:

- Creating physical boundary conditions

- Selectively aggregating molecular compounds within the compartments

- Varying the intracompartmental environment as a stimulus to initialize, control and terminate reactions

- Enabling intercompartmental exchange and feedback mechanisms for interlinked reaction cascades

- Releasing or exchanging the molecular compounds

- Characterizing specific molecular compounds prior to and after the reaction (only necessary in artificial factories, but not found in nature)

In a biological picture of a molecular factory, a lipid membrane usually acts as the boundary for the compartment, and the properties of the membrane are crucial to the performance of the factory. A conceptual illustration of such a bio-inspired molecular factory is shown in Fig. 2A and depicts its main structural components. A large range of bio-inspired molecular compartments and factories has been realized, many being based on lipid bi-layers. Examples are vesicle-based microreactors with spatially segregated reaction pathways $^{[1]}$ or the on-demand release of reactants in a vesicle. ${ }^{[2]}$ DNA-nanocage encapsulation has been shown to enhance catalytic activity while increasing stability, ${ }^{[3]}$ whereas constraining an enzyme within a virus allows for single molecule studies of enzymatic behavior. ${ }^{[4]}$ Even more complex systems requiring different compartments for a multi-step enzymatic pathway have also been demonstrated. ${ }^{[5]}$

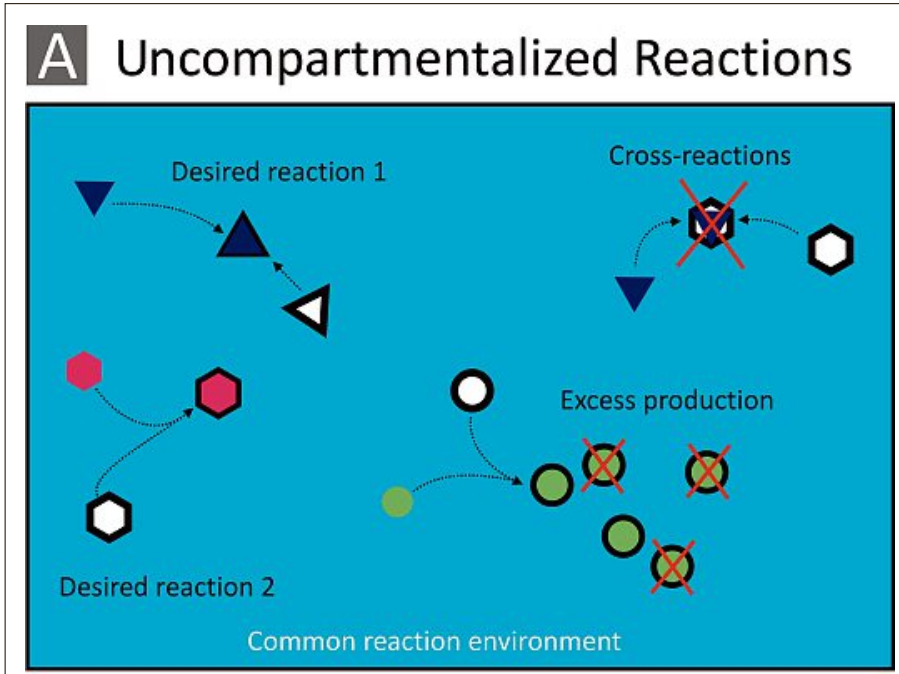

Fig. 1. Comparison of uncompartmentalized versus compartmentalized reactions as well as components required for compartmentalization: A) Besides desired reaction pathways (dark blue: reaction 1, purple: reaction 2 ), undesired side reactions, e.g. cross-reactions among reactants (dark blue) or excess production (green), can take place if pathways are unconstrained and carried out under identical reaction conditions and lack of synthetic selectivity. B) In contrast, compartments with substrate-selective in- and outlets enable reactants to be isolated in corresponding reaction compartments possessing different environments. Exchange and feedback mechanisms enable reactions to be controlled and cascaded. C) Conceptional view of the required components: a physical boundary and selective in- and outlets combined with a controlled micro-environment enable an addressable reaction volume.

\subsection{Solid-state Molecular Factories}

Whereas bio-inspired approaches such as lipid bi-layers or nanocages doubtlessly have their advantages, their size and typically aqueous environment entail a high degree of complexity and large number of molecules while offering limited pathways for downscaling, control over in- and outlets or characterization. In contrast, a rigid, solid-state type of platform allows for smaller volumes by using nanofabrication techniques. This results in a smaller number of molecules and a decrease in the complexity of the system. In addition, the platform allows better control of the environment as well as more stimuli and enhanced characterization methods.

The simplest man-made realization of a solid-state compartment likely is a vial containing a molecular solution, something chemists use on a daily basis. Adding reactants is easily done manually; extracting the products, however, is more difficult and requires chemo-physical methods such 


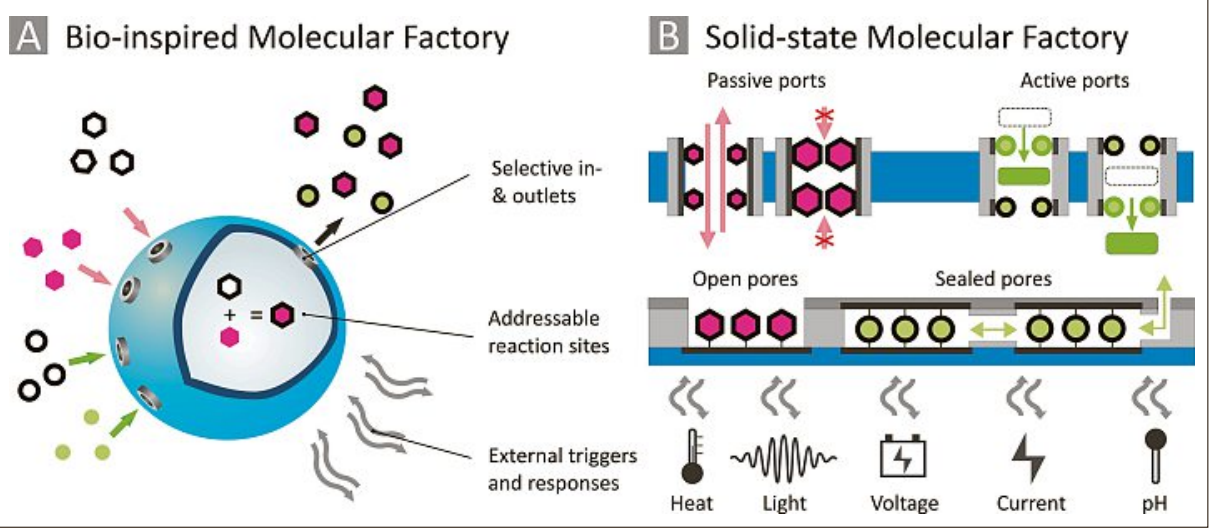

Fig. 2. Basic concept of a bio-inspired (A) molecular factory with structural solid-state (B) components: A) A compartment with in- and outlet ports is established by a membrane structure, enabling reactions to be carried out in an isolated way. B) Structural building-blocks of a solid-state molecular factory based on a silicon platform (blue) allowing selective in- and outlet of reactants by passive and active mechanisms (top row) as well as isolated and individually addressable reaction sites with simultaneous conductance measurement and liquid access through nanochannels (middle row). Electrically conducting parts of the electrodes are indicated in dark gray and can be addressed individually. The platform enables the application of a variety of external triggers or responses such as heat, light irradiation and emission (transparent substrate and electrodes), bias and voltage or electronic current as well as electrochemical triggers, such as ionic currents or $\mathrm{pH}$ (bottom row). Fig. 2A: graphic reproduced from the NCCR MSE homepage, www.nccr-mse.ch

as extraction, filtering, precipitation, crystallization, etc. Further difficulties involve inherent thermal or chemical gradients as well as limited time-resolution in the operations involved. Finally, characterizing the identity of all reactants can be done with optical techniques, e.g. absorption or transmission spectroscopy, an approach that typically involves many thousands of molecules that are probed simultaneously.

With the use of micro- and nanotechnology, the scale of the active volume can be drastically reduced and at relevant volumes of micro liters $(\mu \mathrm{L})$ to pico liters $(\mathrm{pL})$, the field of microfluidics is entered. Here, a significantly different surface-tovolume ratio is present, allowing a major increase in response time when changing the environment or stimuli, for example in terms of thermal management. This size scale is well-covered both in research literature as well as practically in labon-a-chip devices and micro-reactors. ${ }^{[6-8]}$ Further decrease of the size leads to active volumes of femto liters (fL) to zepto liters $(\mathrm{zL})$, where surface and single-molecule effects dominate. The molecular ensemble sizes become billions down to a few thousands, with the potential to reach even the single-molecule level. While the low volumes and surface dominance permit fast feedback mechanisms and high catalytic activities, many conventional detection and analysis methods become impractical owing to the low amount of material and decreasing signal-to-noise ratios. Notwithstanding, monitoring and detection at this scale would enable a very profound understanding of the underlying processes and mechanisms as well as ex- cellent control over the reaction by appropriate stimuli.

To address these issues, we leverage the most recent advances in the field of molecular electronics where electrical measurements are performed on molecular monolayers or even single molecules. When using tailored molecules, conductance measurements become very sensitive to a variety of parameters, such as environmental $\mathrm{pH}$, temperature, light, presence of other compounds, in-site reactions, etc. Furthermore, electrical measurements are fast, can be multiplexed and integrated with semiconductor technology. As such, the possibility to monitor and manipulate different chemical processes in very small compartments allows for a large range of experiments while simultaneously enabling a significant technological payoff in terms of novel device concepts which can directly be integrated using conventional semiconductor technology.

Hence, our goal during the entire project duration of the NCCR MSE is to create a solid-state platform with compartments down to zL volumes, so-called 'nanothe length-scale of a single-molecule and that can be used for a variety of tasks, as sketched in Fig. 2B. In particular, this architecture enables the main components of a molecular factory to be created:

\subsubsection{Controllable In- and Outlets}

Based on tailored molecular building blocks that are electrically contacted in a nanopore, passive in- and outlet ports can be implemented. This function is enabled by the intrinsically switchable properties pores', whose scale in one dimension is on of the building blocks, e.g. the hydrophobic to hydrophilic change of the ferrocene to ferrocenium-ion transition or changes in terms of the molecular volume occupied within the pore (Fig. 2B). Cascading two or more of such ports allows for a liquid cargo to be actively shuttled and even intermediately characterized. The ports can be implemented either in a vertical 'pore-in-membrane' fashion, as depicted in Fig. 2B, or in a horizontal orientation inside a channel.

\subsubsection{Addressable Nanopore \\ Reaction Sites}

Individually addressable nanopores can be kept at different electrochemical potentials with respect to an electrolyte, allowing for selective assembly of different compounds at different sites on the same chip and even in the same channel using, e.g., electrochemically active anchor groups (Fig. 3A). Such open nanopores containing molecular assemblies furthermore offer the possibility to modify the local electrochemical potential by the electrical bottom electrode, a mechanism which can be used for a number of purposes, the simplest being the initialization of a chemical reaction via the electrochemical potential, which may happen in an open pore on the surface of a chip, or an open pore inside a nanofluidic channel.

\subsubsection{External Triggers, Response \\ Sensors and Feedback Mechanisms}

Sealed pores, where the molecules of interest are sandwiched between two electrodes (Fig. 3A), offer a variety of applications where the conductance of the molecular layer can be used as a sensitive probe. Simple experiments allow a direct probing of the response of the molecular film to different triggers, such as heat, light or voltage, and allow the monitoring of conformational changes, oxidation states, etc. This enables the characterization of a molecular film under controlled conditions. Further by adding nanofluidic access, this type of device (Fig. 3B) can be used as a nanometer-scale sensor for specific chemical compounds, electrochemical potential or other such quantities, an integral element in creating feedback loops to control a reaction.

\section{Solid-state Nanopore Architecture}

The above-mentioned primary building block of our platform is the so-called 'nanopore'. It primarily consists of two electrodes separated by a dielectric, with a pore of variable size forming the lateral constraint for a molecular monolayer spanning the space between the two 


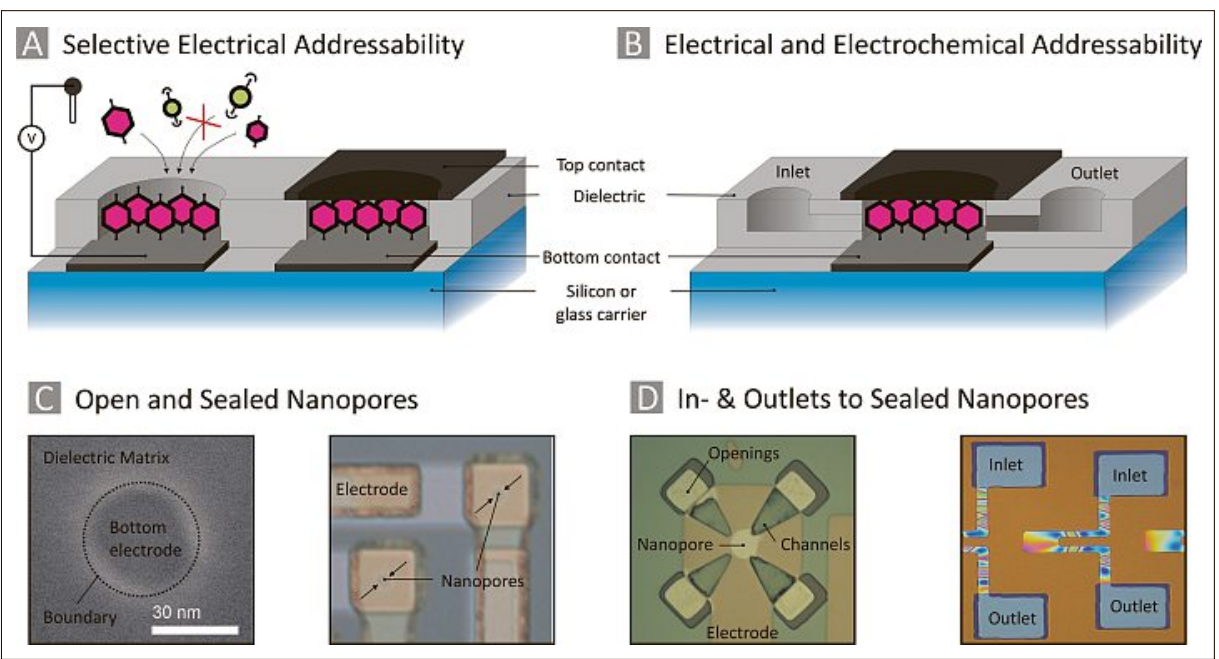

Fig. 3. Cross-sectional view and experimental realization of open and sealed nanopores for electrical and electrochemical studies: Nanopore compartments are etched into a dielectric membrane without $(A)$ and with embedded nanochannels $(B)$, exposing a bottom electrode. The electrical addressability of the electrode enables selective assembly. C) Scanning electron microscopy picture of an open nanopore (left; low contrast as the dielectric layer is only $12 \mathrm{~nm}$ thick) and optical microscopy picture of sealed nanopores (right) with bottom and top electrodes that appear to be semi-transparent owing to the use of $30 \mathrm{~nm}$ thin metal layers. D) Single open nanopore with embedded nanochannels in a quadrant orientation with top access to the electrochemical environment (left) and several micrometer long channels embedded in the oxide matrix after selective dry etching of the buried channels (right). The interference patterns indicate a fully cleared channel.

electrodes, as shown in the middle row of Fig. 2B. Fabricating such a device layer by layer in a vertical fashion permits fabrication methods developed for micro- and nanoscale electronics to create a device where the separation of the electrodes is on the single nm level or beyond, tailored to match the length of the molecular compounds.

\subsection{Fabrication Methods for Nanopores}

Although our architecture is inspired by work done on mono-layer molecular electronics devices, one of our main goals is to maintain compatibility with semiconductor mass fabrication. We therefore avoid exotic or serial fabrication processes as well as gold, a material often used for electrodes but incompatible with frontend-of-line semiconductor fabrication. A schematic of a solid-state nanopore device is depicted in Fig. 3A and B. The basic building blocks are patterned platinum electrodes and dielectric layers deposited via bottom-up atomic-layer deposition (ALD) or plasma-enhanced chemical vapor deposition (PECVD). Besides silicon substrates, transparent substrates such as glass or sapphire, allow direct optical access as ultrathin bottom electrodes on the order of $10 \mathrm{~nm}$ are still conducting but simultaneously optically semitransparent. The devices may also be fabricated on suspended membranes in a flow geometry where the electrodes are placed on the sides, as depicted in Fig. 2B. Patterning the pores into the dielectric can be done using dry and wet etching techniques or a combination of both. The exposed electrodes can act as selective, electrically or electrochemically addressable assembly site, electrical probes or charge-separation units. Fig. 3C shows a scanning-electron microscopy image of an open pore, demonstrating the feasibility of pore diameters on the $30 \mathrm{~nm}$ scale, as well as an optical microscopy image of two closed micrometer-sized pores. By using a template-stripping method described elsewhere, ${ }^{[9]}$ we are further able to combine ultra-flat platinum bottom electrodes with a surface roughness better than $0.3 \mathrm{~nm}_{\mathrm{RMS}}$ over $1 \mu \mathrm{m}^{2}$ with the nanoto micrometer-sized pores in the dielectric matrix, guaranteeing a high-quality of the self-assembled molecular mono layers.

Sealed nanopores are interesting from an electronic and chemical point of view as the molecules are no longer exposed to oxygen or moisture, allowing more complex and reactive molecules to be used. In the context of the NCCR MSE, however, we need physical access to the molecular mono layer, in terms of gaseous, ionic or even molecular-compound exchange. This can be achieved by adding nanometersized channels, as schematically shown in Fig. 3B, where in- and outlets allow for diffusive access to the molecular layer. First steps in this direction were already taken, using a sacrificial layer to pattern the channels, with the latter subsequently being removed via selective dry etching. Fig. 3D shows optical microscopy images of an open pore with four triangular channels (left), with the sacrificial material still in place, as well as etched channels connecting an inlet to an outlet (right), with the buried, released channel causing an interference pattern.

\subsection{Current State: Electrical Characterization of Alkane-dithiol Mono Layers}

Due to the interest in electronic devices based on intrinsic molecular functionalities, the development of methods to electrically contact monolayers of molecules has been the subject of intense investigation and a large variety of strategies has been suggested. A number of challenges need to be addressed here, the first being the fabrication of a smooth bottom electrode for growing high-quality films. This smoothness needs to be maintained throughout the dielectric deposition and etching steps. Second, the deposition of the top electrode poses a significant challenge as the integrity of the molecular film has to be maintained. Ideally, the fabrication flow should further be compatible with standard mass fabrication techniques, avoid slow serial processes, and have a large device yield, all while maintaining the desirable properties of the molecular film.

Different methods have been proposed for the deposition of the top contact, ranging from direct[10] and indirect ${ }^{[11]}$ physical vapor deposition (PVD), conducting polymer $^{[12,13]}$ or graphene protective layers ${ }^{[14]}$ to more exotic deposition methods, such as micro-contact printing ${ }^{[15,16]}$ or single gold crystals. ${ }^{[17]}$ Each of these approaches has drawbacks, be it low yield, significant added series resistance, or a serial fabrication approach preventing cost-efficient large-scale integration. We fabricate the top contact in a two-step process, where a protective layer is first deposited from solution. ${ }^{[18,19]}$ This layer not only provides a well conducting contact to the molecules, but also protects the film during the subsequent deposition of a regular metal layer via $\mathrm{PVD}$, providing the compatibility with standard processes. The conformal nature of this protective layer creates contacts to films even in sub-30 $\mathrm{nm}$ pores in thicker (10-20 nm) dielectrics, where the aspect ratio between dielectric thickness and nanopore diameter approaches 1:1.

While the solid-state nanopore is conceptually simple, a review of the state of the art shows that such a platform is non-trivial in its actual fabrication. In a first step, we therefore aim to reproduce and improve on the results obtained with alkanedithiols, which is a passive but highly sensitive and modular test bed. Consisting of an alkane backbone with one (alkane monothiol) or two anchor groups (alkane dithiol), these compounds are considered a prototypical system for transport and are 
therefore most often used to validate novel molecular electronics platforms. Different chain lengths are readily available commercially and allow a systematic study of the length dependence of the tunneling current, enabling a cross-platform comparison as well as proving the sensitivity of the platform to the type of molecule. A sketch of the electron transport study through octane dithiol is depicted in Fig. 4A, where the electrons schematically tunnel non-resonantly through the backbone of the molecule. We achieve a high yield using our method as illustrated in Fig. $4 \mathrm{C}$, where the device behavior (molecular non-linear current-voltage characteristics, shorted or open nanopores) is plotted for pore diameters smaller and larger than 1 $\mu \mathrm{m}$ for the example of octane dithiol (C8). We observe a number of open nanopores for smaller diameters owing to imperfect etching rather than missing contacts to the molecular compounds, whereas for larger devices the yield is better than $90 \%$, even including $100 \mu \mathrm{m}$ pore diameters. Details of this study are published elsewhere. ${ }^{[18]}$

\section{Components for Molecular Factories}

Our final goal in the NCCR MSE, however, is establishing a much more complex molecular factory based on a cascade of reaction compartments, linked through different types of interconnects, ranging from electrical to optical, ionic to mass flow, and controlled via sensors and feedback loops. The generic nanopore platform will support this goal throughout the project, first by allowing for each molecular component to be characterized individually under controlled conditions, enabling an in-depth understanding of the underlying molecular reaction mechanisms. A next step is interconnecting compartments whose interactions can be controlled. An advantage of the nanopore concept is its flexibility, as the platform can be used in different ways, depending on the molecular compounds and geometryrealized. We therefore aim at studying triggers, implementing sensors and realizing interconnections parts, each requiring molecular compounds tailored to the individual tasks.

\subsection{Requirements for Molecular Compounds}

To obtain devices with intrinsic functionalities that respond to or create, e.g. variations in the bias or conductance, optical absorption or emission, the chemical or electro-chemical environment, tailored functional molecules are required. The nanopore platform imposes several constraints on these molecules, mainly

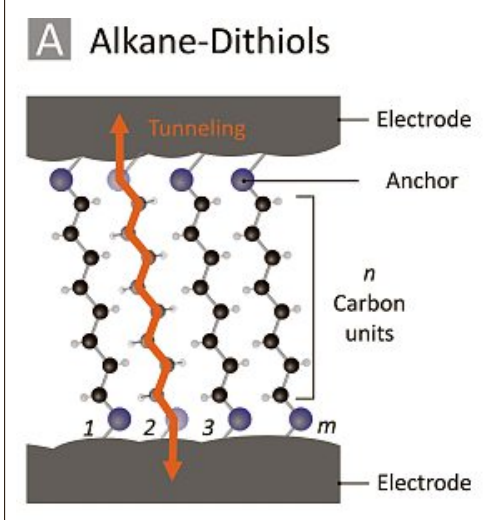

B Light-Sensing

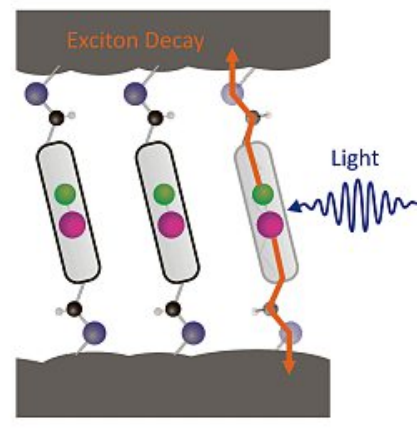

Sensing
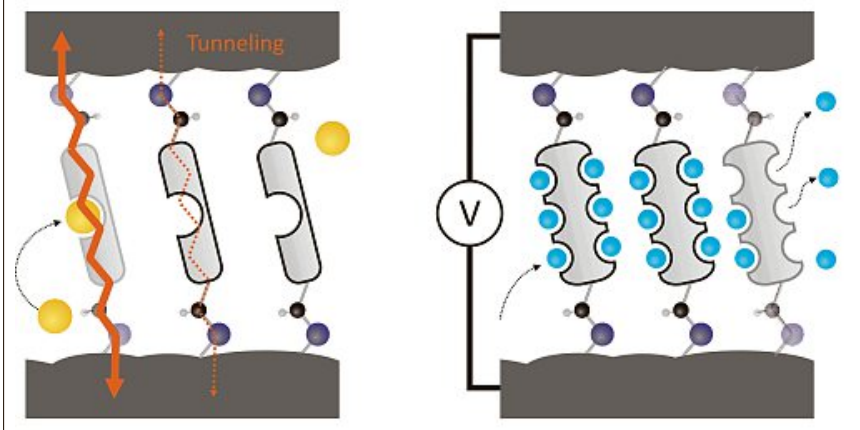

E Device Yield C8 $\mathrm{d}_{\text {pore }}<1 \mu \mathrm{m}$ F Device Yield C8 $\mathrm{d}_{\text {pore }}>1 \mu \mathrm{m}$

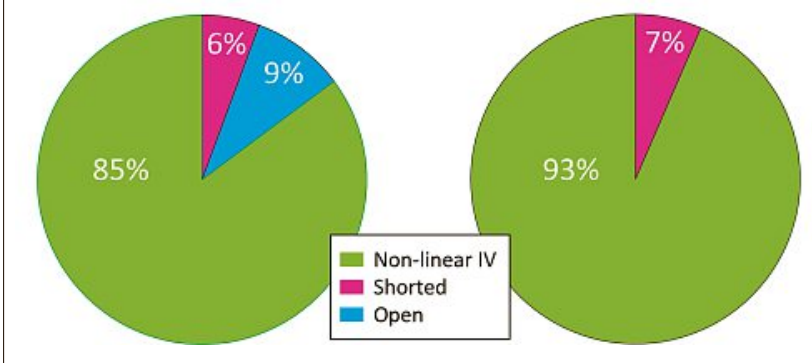

Fig. 4. Electrical nanopore test devices using alkanedithiols and other tailored compounds: A) Alkanes-dithiols are self-assembled into nanopores and contacted by a top electrode to enable conductance measurements. By exchanging the alkanedithiols by more functional molecules, light-sensitive or light-radiating devices (B), analyte-specific sensing devices (C) as well as storing or releasing devices can be established (D). Diameter specific analysis of currentvoltage curves in a device fabricated with octane dithiol (C8) (E and F). For pore diameters smaller or larger than $1 \mu \mathrm{m}$, the number of shorts is in the single percent range. For pores with diameters below $100 \mathrm{~nm}$, we additionally obtain open contacts due to imperfect etching reducing the yield (E). Nevertheless, the overall yield of nonlinear current-voltage curves is better than $85 \%$ for 3500 devices. that they have to be equipped with anchor groups to bind to the electrodes, be it simple thiols or more functional end groups designed for site-specific, deterministic assembly. For closed pores with electrical contact to the molecules, a second crucial requirement is that the molecules self-assemble into densely packed monolayers with a quality sufficient to prevent metallic filaments from penetrating the monolayers and thereby reducing the device yield.

Initially we will be using large-scale and homogeneous self-assembly to achieve films of identical molecules in all nanopores of a device. But a short term goal is the implementation of methods to selectively deposit different types of molecules into specific pores, a prerequisite for more complex circuits with enhanced functionality. Ideally, this selective assembly is electrically triggered, as material contrast - even though technically feasible - provides insufficient selectivity for molecular assemblies. Our focus is on the use of electrochemically addressable protection groups, which allow for different molecules to be immobilized in separate pores on the same chip. Fig. 3A conceptionally shows how the electrochemical potential of a specific nanopore can be varied to allow compound-specific release of the anchor-group protection unit.

\subsection{External Triggers and Feedback Mechanisms}

Two of the main elements that permit the operation of a complex molecular factory are triggers and sensing mechanisms, which enable initiating, controlling and terminating a reaction. Fig. $2 \mathrm{~B}$ depicts a variety of external triggers that can interact with the nanopore platform:

- Heating or cooling of the nanopore (e.g. by heating or cooling of the entire device or by using local microscopic heaters and/or Peltier elements to only address specific nodes) 
- Optical irradiation of individual nanopores using focused light (laser) sources when using transparent carriers (e.g. quartz) in conjunction with semitransparent metal electrodes (5 $\mathrm{nm}$ Pt films still transmit $50 \%$ of the incident light)

- Change of the electrochemical evironment (e.g. $\mathrm{pH})$ and ionic exchange

- Current or bias variation over the nanopores

Simple characterization experiments involve applying one of the triggers listed and monitoring the resulting change in the transport characteristics of the nanopores. By using tailored molecules, sensors sensitive to specific triggers can be realized, such as light sensors, thermometers or $\mathrm{pH}$ sensors. By basing the sensors on molecules, both small volumes and fast response times may be realized, allowing fast feedback loops. For specific triggers, it may be possible to use the nanopores as electrically controlled stimuli sources themselves, such as light emitters, or as sources for releasing ions, atoms or even molecular moieties. Similarly, the individual electrical addressing of each nanopore enables e.g. local accumulation of reactants based on electrochemical mechanisms. Fig. 4B-D depicts various mechanisms affecting the electrical conduction of a molecule enabling light and analytesensing as well as storage or release.

\subsection{Nanofluidic Interconnects}

An essential part in creating a cascade of individual compartments are the interconnects between individual nanopores. These can be simple nanofluidic channels which allow electrochemical communication between different nanopores via ionic exchange or change in electrochemical potential. This architecture can incorporate both open and closed pores, such that both electrochemically addressable reaction sites and conductance-based sensors can interact via the channels. Complex networks of such pores can be envisioned, where pores filled with different types of molecules are interconnected by such channels, as depicted in Fig. 5A.

Although ionic channels already offer a number of interesting properties to be studied, a cascade of reactors will require channels supporting mass flow, combined with elements such as valves and pumps. With the goal of integrating a number of such devices on a single chip, a long-term goal will be the implementation of such components in an electrically controllable form. Valves may be based on electrically controllable hydrophobicity, change in volume or other mechanisms. ${ }^{[20-22]}$ Once realized, this can be expanded by cascading a number of such valves, resulting in an active pump. Our process for fabricating

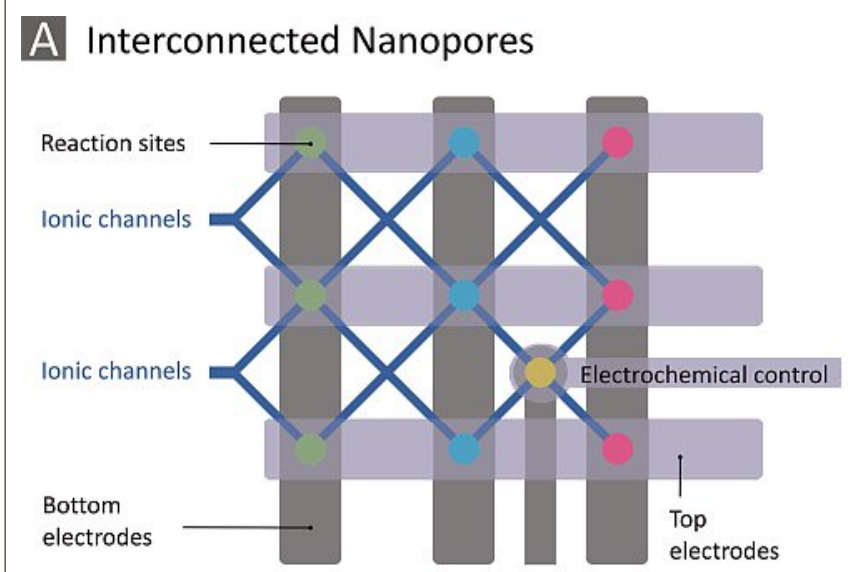

\section{B Fully-integrated Compartments}

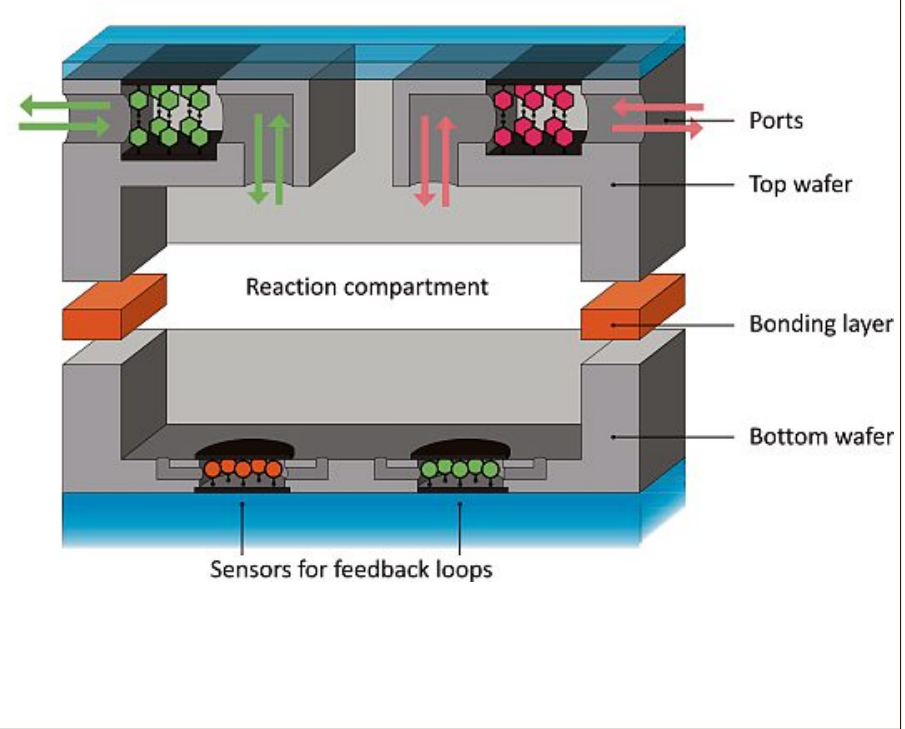

Fig. 5. Upscaling of individual nanopore devices to more complex networks of interconnected nanopores and ultimately to an entire molecular factory: A) Similarly to an integrated circuit, individual compartments of a molecular factory can be studied on a planar Si platform with distributed reaction sites that are interlinked via electrical, electrochemical and optical mechanisms to study the distributed effect of triggers and provide a model for feedback loops. B) By using standard wafer bonding technologies, two wafers with different components and cavities can be bonded together, forming a closed reaction compartment with in- and outlet ports, sensing structures, etc. By fabricating a number of these structures, complex cascades of different compartments combine to form a full nanoscale molecular factory. channels is compatible with placing electrodes at the top and bottom of a channel. Combining this with site-specific deposition of molecules, valves and pumps on the nm scale may be realized in conjunction with functionalized nanopores.

The placement of electrodes at the top and bottom of a nanofluidic channel also opens up the possibility of performing capacitance measurements for impedance spectroscopy. By fabricating the measurement circuits on the same substrate, stray capacitance can be minimized, allowing precise measurements and large frequency ranges, while the low measurement volume increases sensitivity to specific compounds.

\subsection{Small-Volume Feedback- Controlled Reaction Compartments for Cascaded Reactors}

Combining sensors based on molecules with controllable in- and outlet ports sets the basis for a small-volume reactor with feedback control, a schematic shown in Fig. 5B. Using site-specific assembly and wafer-to-wafer bonding, a reaction compartment with the necessary components can be fabricated mainly using standard silicon processes. In particular, the compatibility of our nanoporebased sensors and valves with standard CMOS technology allows for the control circuits to be fabricated on the same wafer, enabling an 'all integrated' approach to feedback-controlled reaction compartments. Furthermore, the small size of the compartments, in principle, enables a cascade of different compartments, where starting materials and intermediate products are selectively reacted towards a complex value-added product. Although a single cascade has a low volume, the small size can be expected to yield faster reactions as well as enable a large number of parallel 'assembly lines' to be run, significantly increasing the expected output.

\section{Summary and Outlook}

A novel solid-state platform for electrically addressable zeptoliter compartments was presented, where one compartment dimension is comparable to the length of a molecule. This platform can be used in a 
variety of geometries, on the one hand, to study and mimic components and processes of biologically inspired molecular factories, while on the other hand allowing the necessary components for a molecular factory to be realized in a silicon platform. We have first demonstrated sensitive conductance measurements on alkane-dithiol self-assembled monolayers, showing the feasibility and achieving high device yields. Such conductance measurements can now be leveraged for investigating the response of molecular films to different stimuli, namely bias, heat, light or electrochemical potential. We plan to investigate different types of molecules for their application as components in a molecular factory, the long-term goal of the NCCR MSE. By interconnecting different compartments with nanofluidic channels, a system with increasing complexity can be realized to study feedback loops between different reaction sites. Our long-term vision furthermore includes electrically controlled valves and pumps in the interconnects, paving the way for fully integrated reaction compartments encompassing in- and outlet ports, sensors and feedback loops for control. By cascading such compartments, a solid-state implementation of a molecular factory can be realized, where the silicon platform enables the factory paradigm to be extended to non-biological products.

\section{Acknowledgements}

The authors acknowledge technical support by M. Tschudy, U. Drechsler, and M. Bürge, as well as strategic support by B. Michel, W. Riess, and A. Curioni. Funding by the SNF via the NCCR MSE is highly appreciated.

Received: April 12, 2016

[1] Y. Elani, R. V. Law, O. Ces, Nat. Commun. 2014, 5,5305 .

[2] P.-Y. Bolinger, D. Stamou, H. Vogel, J. Am. Chem. Soc. 2004, 126, 8594.

[3] Z. Zhao, J. Fu, S. Dhakal, A. Johnson-Buck, M. Liu, T. Zhang, N. W. Woodbury, Y. Liu, N. G Walter, H. Yan, Nat. Commun. 2016, 7, 10619.

[4] M. Comellas-Aragones, H. Engelkamp, V. I. Claessen, N. A. J. M. Sommerdijk, A. E. Rowan, P. C. M. Christianen, J. C. Maan, B. J. M. Verduin, J. J. L. M. Cornelissen, R. J. M. Nolte, Nat. Nano 2007, 2, 635.

[5] V. Noireaux, A. Libchaber, Proc. Nat. Acad. Sci. USA 2004, 101, 17669.

[6] H. A. Stone, S. Kim, AIChE Journal, 2001, 47, 1250.

[7] D. J. Beebe, G. A. Mensing, G. M. Walker, Annu. Rev. Biomed. Eng. 2002, 4, 261.

[8] G. M. Whitesides, Nature, 2006, 442, 368.

[9] G. Puebla-Hellmann, M. Mayor, E. Lörtscher, Nanotechnology (IEEE-NANO), 2015 IEEE 15th International Conference, July 2015, pp. 1197-1201.

[10] C. Zhou, M. R. Deshpande, M. A. Reed, L. Jones, J. M. Tour, Appl. Phys. Lett. 1997, 71, 611.

[11] H. Haick, M. Ambrico, J. Ghabboun, T. Ligonzo, D. Cahen, Phys. Chem. Chem. Phys. 2004, 6, 4538

[12] H. B. Akkerman, P. W. M. Blom, D. M. de Leeuw, B. de Boer, Nature 2006, 441, 69.

[13] A. B. Neuhausen, A. Hosseini, J. A. Sulpizio, C. E. D. Chidsey, D. Goldhaber-Gordon, ACS Nano, 2012, 6, 9920.

[14] G. Wang, Y. Kim, M. Choe, T.-W. Kim, T. Lee, Adv. Mater. 2011, 23, 755.

[15] A. Vilan, D. Cahen, Adv. Funct. Mater. 2002, 12, 795.

[16] Y.-L. Loo, D. V. Lang, J. A. Rogers, J. W. P. Hsu, Nano Lett. 2003, 3, 913.

[17] R. Boya, D. Jayaraj, G. U. Kulkarni, Chem. Sci. 2013, 4, 2530.

[18] G. Puebla-Hellmann, M. Mayor, E. Lörtscher, to be submitted, 2016.

[19] G. Puebla-Hellmann, M. Mayor, E. Lörtscher, 'Method to Contact Self-assembled Monolayers of Molecules', Patent Application Pending, 2016.

[20] M. G. Pollack, R. B. Fair, A. D. Shenderov, Appl. Phys. Lett. 2000, 77, 1725.

[21] S. K. Cho, H. Moon, C.-J. Kim, J. Microelectromech. Systems, 2003, 12, 70.

[22] H. Liu, S. Dharmatilleke, A. A. O. Tay, Microsystem Techno. 2009, 16, 561. 